\title{
Research Method of Data Deduplication Backup System
}

\author{
V.Sathiya Suntharam, Sheo Kumar, Chandu Ravi Kumar
}

\begin{abstract}
In this paper, we've got studied cutting-edge-day backup systems and used a singular technique which facilitates in decreasing the fragmentation trouble.

The winning backup tool have lumps of every backup which is probably bodily scattered, which ends up in a very demanding fragmentation trouble.

Fragmentation offers upward thrust to 2 kinds of fragmented containers which is probably sparse and out-of-order boxes. Sparse container exacerbates the device simple ordinary overall performance on both restore and rubbish series. Out-of-order packing containers talk to the ones containers which can be accessed commonly at some point of a repair. As a way to lessen the fragmentation trouble we use the antiquity acquainted set of guidelines and stockpile aware filter out. Those help in figuring out the sparse further to the out of order discipline.
\end{abstract}

Key terms: Fragmentation, Sparse, deduplication, containers, chunks

\section{ADVENT}

Deduplication has already been a warm difficulty count because it efficiently receives rid of the duplicates. The effectiveness of such approach in lowering whenever had to perform backups and garage place required to keep them has been drastically defined [1][4][13].

Inside the civilized backup structures deduplication plays a essential position as it has the capability of enhancing the garage wellknown performance. In workout, deduplication has become definitely taken into consideration virtually one of critical capabilities of backup device [2][5].

Traditionally, the overall preferred performance of deduplication tool is described with the aid of manner of way of deduplication ratio, maximal

Examine and write overall overall performance. Packing containers are the vital unit of have a have a look at and write operations. Containers are steady-sized shape wherein small and variable sized chunks are controlled. Throughout initial backup there may be no fragmentation trouble as the reproduction chunks are not removed however in the course of a couple of backups, the chunks grow to be physical scattered in various boxes, that is known as fragmentation [3] [12].

Fragmentation is a herbal by-product of deduplication. It motives harm in methods- first of all, the fragmentation drastically decreases the repair usual performance. On every

\footnotetext{
Revised Manuscript Received on September 10, 2019.

V.Sathiya, Department of CSE, Professor, CMR Engineering College, Hyderabad, Telangana,India.

(E-mail: sathiya4196@gmail.com)

Dr.Sheo Kumar, Department of CSE, HOD , CMR Engineering College, Hyderabad, Telangana, India.

(E-mail: csehod@cmrec.ac.in)

Prof.Chandu Ravi Kumar, Department of CSE, CMR Engineering College, Hyderabad, Telangana, India.

(E-mail: chanduravikumar@gmail.com)
}

occasion the patron deletes the expired backup, the invalid chunks are bodily scattered a number of the precise bins due to fragmentation.

Now, the rubbish collection [6] tries to choose out out valid chunks and the field maintaining legitimate chunks. This could become very time ingesting. After the approach of identification the legitimate chunks are copied to new bins [7][8] and they may be reclaimed.

On monitoring, we be aware that fragmentation is of sorts, Sparse packing containers and Out of order packing containers, which in my opinion have horrible consequences. Sparse containers exaggerates have a have a look at operations at the identical time as Out-of-order field outcomes the restore common universal overall performance. The chunks in sparse bins are never accessed in some unspecified time in the future of a backup, and the chunks in out-of-order discipline are accessed continuously.

The prevailing strategies [9][11][14] supply hobby to a small a part of the backup, and pick out out out the fragmented chunks within the small element. Here, the primary drawback is that the out-of-order bins are misinterpreted as sparse bins and as a quit cease end result the sparse containers are not identified, which leads to a totally constrained advantage in the repair installed usual performance.

The purpose within the returned of the evolution of [AAA]Antiquity acquainted set of guidelines are the observations located sooner or later of two consecutive backups, wherein the facts collected at some stage in initial backup is used for enhancing the following backup. AAA overcomes the drawbacks of the prevailing approaches thru rewriting the duplicate chunks inside the sparse container decided within the previous backup after which makes a vote of the growing sparse field to rewrite them inside the subsequent backup. To beautify the repair regular overall performance, we extend the hybrid scheme and it ever decreases the deduplication ratio to a very little quantity.

The AAA reduces the sparse containers, due to which the approach of figuring out legitimate chunks within the direction of the rubbish series isn't essential. An set of rules referred to as Cask-display display set of pointers(CMA) which identifies legitimate bins as opposed to legitimate chunks and decreases the overhead. The the rest of the paper is ready as follows.

The subsequent section i.E.,

Phase 2 gives the literature survey.

Segment 3 appears nearer on the evolution and outline of the fragmentation hassle, 
Segment 4 gives records regarding the two training of fragmentation, sparse and out-of-order packing containers.

Phase 5 concentrates on the solution of decreasing the fragmentation problem [AAA] Antiquity acquainted algorithm

Section 6 appears into the scheme used for reinforcing the repair standard overall performance.

Section 7 gives with the CMA [10].

Sooner or later, in segment 8 we finish our paintings.

\section{LITERATURE SURVEY-}

Name: keeping off the disk bottleneck in the information region de duplication file tool.

Writer: Benjamin Zhu, Kai li.

Twelve months: 2008

Description: This paper describes 3 strategies hired within the manufacturing facts area de duplication report device to relieve the disk bottleneck. The ones techniques encompass: (1) the precis Vector, a compact in-memory statistics shape for identifying new segments; (2) circulate-informed section layout, a facts layout approach to enhance on-disk locality for sequentially accessed segments; and (three) Locality Preserved Caching, which maintains the locality of the fingerprints of duplicate segments to acquire immoderate cache hit ratios. Together, they are capable of cast off $99 \%$ of the disk accesses for de duplication of real global workloads. Those strategies permit a cutting-edge -socket dual-center device to run at $90 \%$ CPU usage with simplest one shelf of 15 disks and accumulate one hundred $\mathrm{MB} / \mathrm{sec}$ for single-movement throughput and $210 \mathrm{MB} / \mathrm{sec}$ for multicirculate throughput.

Name: "improving restore tempo for backup systems that use inline bite-based completely deduplication".

Creator: Mark Lillibridge, KaveEshghi.

Twelve months: 2003

Description: gradual recovery because of chew fragmentation is a immoderate trouble managing inline chunk-based statistics de duplication systems: repair speeds for the maximum present day backup can drop orders of significance over the existence of a device. We check three techniques - developing cache duration, situation capping, and the usage of a earlier assembly vicinity-for alleviating this problem. Container capping is an ingest-time operation that reduces bite fragmentation on the rate of forfeiting some de duplication, on the identical time as the use of a forward meeting place is a contemporary restore-time caching and prefetching method that exploits the right information of destiny chunk accesses available on the same time as restoring a backup to lessen the amount of RAM required for a given level of caching at repair time.

Call: Restoring de-duped information in deduplication systems.

Writer: W. Curtis Preston.

Year: 2005

Description: facts is a manner to reduce storage desires via removing redundant facts to your backup surroundings. First rate one reproduction of the records is retained on garage media, and redundant statistics is changed with a pointer to the proper statistics replica. Dedupe technology typically divides information gadgets in to smaller chunks and uses algorithms to assign each facts chunk a hash identifier, which it compares to formerly stored identifiers to determine if the statistics chunk has already been stored. A few companies use delta differencing era, which compares current-day-day backups to previous facts on the byte stage to put off redundant facts.

Name:A scalable height throughput exacts deduplication approach for community backup offerings.

Author: J. Wei, H. Jiang, ok. Zhou, and D. Feng.

12 months : 2007

Description: De duplication has been notably applied in disk-based totally definitely secondary storage systems to decorate place basic performance. But, there are disturbing situations going thru scalable excessive-throughput de duplication garage. The primary is the reproduction-studies disk bottleneck due to the big duration of records index that generally exceeds the to be had RAM location, which limits the de duplication throughput. The second is the storage node island effect because of duplicate facts amongst multiple storage nodes which is probably difficult to dispose of. Present strategies fail to without a doubt remove the duplicates on the same time as simultaneously addressing the demanding conditions.

Name:decreasing effect of facts fragmentation due to inline deduplication.

Author: M. Kaczmarczyk, M. Barczynski, W. Kilian.

12 months : 2012

Description: Deduplication results necessarily in data fragmentation, due to the fact logically non-forestall data is scattered within the direction of many disk places. In this paintings we reputation on fragmentation due to duplicates from preceding backups of the equal backup set, considering that such duplicates are very common due to repeated whole backups containing loads of unchanged information. For systems with in-line dedup which detects duplicates for the duration of writing and avoids storing them, such fragmentation causes facts from the extraordinarily-current backup being scattered all through older backups. As a give up result, the time of restore from the current-day-day backup may be drastically expanded, every so often greater than doubled.

\section{FRAGMENTATION TROUBLE}

Fragmentation trouble slows down the device traditional general overall performance for repair and garbage series.

We are able to say that on the identical time as performing consecutive backups, the Fragmentation hassle will upward thrust up. Fig.1 suggests an example wherein restoring the backups will have an effect at the machine usual ordinary performance. The primary backup includes 10 chunks of information every diagnosed with the resource of someone. The reproduction chucks, adequate and N, are recognized with the useful useful resource of deduplicating the go together with the flow called self referred chunks. In this case, all of the right chunks are stored in first 3 boxes and a easy is allocated to second half of of of of the $1 /$ three subject. It makes use of 3-place-sized LRU cache and is 
wanted to examine 5 bins thru default for restoring first backup.

The second one backup moreover includes 10 chunks, 7 of which may be duplicates of first backup. The 3 final chunks are saved in new discipline. To repair 2nd backup, by way of manner of default 9 packing containers are to be examine. As a quit end result, we're in a role to mention that $2 \mathrm{~d}$ backup would require reading of four greater chunks than that of first backup. This will worsen the restore normal performance of the device.

After deleting the primary backup, three chunks $(\mathrm{M}, \mathrm{P}, \mathrm{R})$ becomes invalid however we can not declare them proper away as there exist excellent chunks which can be used by the second backup. Gift artwork makes use of the offline field merging operation [15],[16].The merging could have a examine and copy the few legitimate chunks into new boxes. Therefore, this will bring about a time ingesting segment inside the rubbish collection [14].

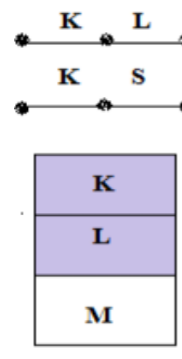

$\mathbf{I}$

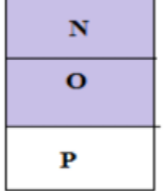

II

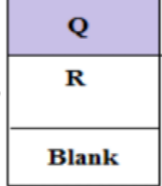

III

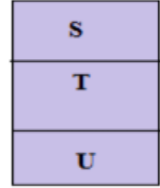

IV

\section{Fig.1 Example}

\section{KIND}

There are two styles of fragmented container, sparse vicinity and out-of-order box.

\section{SPARSE field:}

After deleting the number one backup, we require a merging operation to mention the valid containers. This form of boxes worsen tool regular standard overall performance on restore and rubbish series. As an instance go through in mind Fig.1, best bite $Q$ in $1 /$ three area is referenced with the beneficial useful resource of the use of the second backup, it really is inefficient at the same time as restoring the second one backup. The packing containers whose utilization is smaller than a predefined usage threshold, this is $50 \%$, the sector is taken into consideration as a sparse location. To diploma the general sparse diploma of the backup, we use the not unusual appoint of all the bins associated with a single backup. If sparse boxes pre-fetches a area of fifty\% usage, it will gain $50 \%$ of the maximum garage bandwidth, as $50 \%$ of the chunks in that area are in no manner accessed. Those chunks would require slots in the restore cache, with the intention to lower the size of to be had cache. Therefore, if the sparse packing containers are reduced, the restore common overall performance may be advanced. It is likewise determined that garbage series reclaims high-quality little region with none more mechanisms, which include offline merging sparse difficulty. The merging operation suffers from a normal common overall performance problem. Because of this, we require a greater inexperienced technique to take a look at and duplicate the valid chunks into new containers.

\section{OUT-OF-ORDER box:}

A vicinity that is accessed very often in some unspecified time within the future of a repair is apprehend as out-oforder subject. As proven in Fig.1, whilst restoring the second backup 4th box is accessed 3 times, this may make it a out-of-order field. Restoring each bite inside the container will surrender give up quit end result to cache pass over that decreases restored overall performance.

Out-of-order packing containers together with self referenced chunks will complicate the trouble. If the get right of entry to time of self referenced chunks is a lot less, then it'll result in a better repair common average performance. While if the get proper of access to time is more, repair traditional regular common overall performance decreases. As an instance, in Fig.1, chunk 'good enough' will decrease the restore regular usual overall performance and chew ' $\mathrm{N}$ ' will increase the repair enormous basic overall performance. As the two accesses to chunk ' $N$ ' get up near in time.

The impact of out-of-order region on repair performance is predicated upon on repair cache. For each repair, there exist a minimal cache length, called cache threshold, that is required to acquire the maximum repair conventional basic universal overall performance. The smaller the cache duration than the cache threshold, the greater is the effect of out-of-order container at the repair fundamental normal usual overall performance. A sufficiently massive cache can remedy the trouble but the memory is steeply-priced. Consequently, it is unaffordable. Out-of-order container have no horrific effect on garbage collection.

\section{ANTIQUITY FAMILIAR ALGORITHM[AAA]:}

While the backup device is initiated, AAA masses the identity's of all of the sparse bins (already sparse in the previous backup similarly to in the modern-day backup) to convey together the in-memory form referred to as as Sinherited. At some level in the backup, AAA rewrites all the replica chunks whose identification exists in Sinherited.In real, HAR maintains in-memory systems, Ssparse and Sdense (present within the accrued records ), to build up the identification's of the growing sparse containers.

The Ssparse lines the containers whose utilizations are smaller than the utilization threshold. The Sdense statistics the boxes whose usage exceeds the utilization threshold Essentially, the two systems consist of usage facts, and each file includes the sector identity plus the current usage of the 
box. After the backup is completed, AAA replaces the identification's of the vintage inherited sparse containers with the identification's of the growing sparse containers in Ssparse. Therefore, the Ssparse becomes the Sinherited of the following backup.

A large quantity of growing sparse containers shows that we've got got had been given were given many fragmented chunks to be rewritten within the next backup. AAA uses a rewrite limit to decide whether or not there are too many sparse containers in the in-reminiscence structures. AAA calculates the rewrite ratio for the backup. It first calculates the expected period of rewritten length for each box through multiplying utilization with size of the sector. Then predicted rewrite ratio (described as the dimensions of rewritten records divided via the backup period) is calculated. If the expected ratio exceeds the predefined restriction AAA receives rid of the record of the maximum important usage in Sdense..

Whilst in contrast to the alternative in-reminiscence systems Sinherited is negligible. The rest consumes extra reminiscence because it wants to show show display screen all boxes related to the backup. There's a compromise in AAA. On every occasion there may be a immoderate usage threshold, there are extra bins which can be taken into consideration as sparse, and consequently the backups are of better common utilization and restore standard normal performance however worse deduplication ratio.

\section{HYBRID SCHEME}

Antiquity acquainted set of pointers(AAA) requires massive area sized restore cache to carry out capping because of the truth virtual tool snap shots include a huge form of self-references which also can moreover make the problem created thru the out-of-order boxes even worst considering that almost big such big repair cache is unaffordable because of concurrent repair processes.

Hybrid scheme is brought into the frame for the reason that maximum of the chunks rewritten through the winning algorithms belong to the out-of-order packing containers and through manner of the use of Hybrid scheme we will make use of each the prevailing rewriting algorithms (eg: CBR[17] and capping [5]) further to AAA. Because of the truth the winning rewriting algorithms have lots an awful lot less knowledge about the restore cache they may be willing to restore many useless out-of-order packing containers Hybrid scheme is used because it's a straight away beforehand method.

The easy combination a few of the Hybrid scheme and each AAA and gift rewriting algorithms permits in reducing deduplication of data. As a result we need to optimize the prevailing rewriting algorithms to avoid vain deduplication and decrease fragmentation.

Permit us to take a example to recognize the method of the sample backup system greater actually.

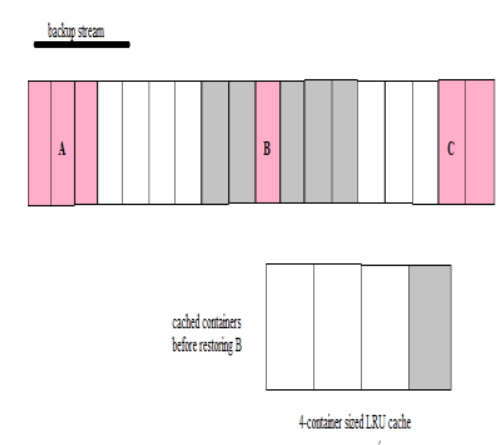

Fig:2 4-box-sized LRU cache

The pink colored area is the out-of-order location with 8 chunk sized rewriting buffer. The chunks A and C are beyond the scope of the rewriting buffer so the chunk B is end up rewritten. The chew $B$ will not negatively have an impact at the restore performance as it's miles related to four-container-sized LRU cache and the purple boxes is been consistent with fetched for storing $\mathrm{A}$ and will live within the cache at the same time as B is restoring. To keep away from any forms of storage usual overall performance problems B shouldn't be rewritten extra times.

To optimize the winning rewriting algorithms Cacheconscious-clean out (CAF) is evolved. Our essential purpose is to have a observe whether or not or not or now not or not the collection of the restoring chunks is much like the collection of rewriting them in a few unspecified time inside the future of them at some point of the backup technique. So while a LRU repair cache with a predefined period is given we are capable of $\mathrm{u}$. S . Its runtime country at some point of the backup without trouble due to the truth CAF simulates the LRU restore cache throughout backups by means of the usage of the usage of the use of using the to be had container identification's inside the backup tool float. To actually understand the technique take a look at the above example i.E (fig:2) at the equal time as the bite B is subsidized-up, CAF is aware about the pink field ought to stay in the repair cache due to the reality we're the use of the four-regionsized LRU cache. CAF doesn't approve the request of rewriting B which allows in improving the present rewriting algorithms in terms of every storage performance and repair overall performance.

\section{CASK -SHOW SET OF RULES}

To reduce the metadata overhead of the rubbish series, we further suggest a Cask- show set of regulations (CMA) to come to be privy to valid boxes in area of valid chunks. The triumphing rubbish series schemes rely upon merging sparse containers to reclaim invalid chunks within the bins. In advance than merging, they ought to choose out invalid chunks to determine utilizations of boxes, i.E, reference 
manipulate . Present reference control techniques [12]-[14] are always cumbersome because of the existence of big portions of chunks. To avoid this Cache-conscious clean out (CAF) make the most cache understanding. With the help of $\mathrm{CAF}$, the hybrid scheme notably improves the deduplication ratio without decreasing the repair everyday average performance. CAF can be used as an optimization of gift rewriting algorithms.

AAA hundreds the identification's of all of the sparse boxes to bring collectively the in-memory form at the equal time as $\mathrm{CMC}$ is superior in a way wherein it permits to decide the invalid and valid containers within the backup device. The approach takes location in the FIFO (first in first out) order, wherein the oldest backup is deleted first just so the man or woman has most time to recheck the facts.

CMA keeps a field occur for each dataset. All the id's of the packing containers present are recorded and they will be saved collectively with a backup time, which shows that precise datasets modern day backup associated with that box.

All of this is stored in the location seem. Even as we need to delete the oldest backup of the database CMA hundreds the box display up into the memory. CMA is considered to be fault tolerant and recoverable because of the truth if the data list gets corrupted, we are able to right away get better the information via traversing manifests of all the associated datasets.

\section{FORESTALL}

On this paintings we've got defined facts fragmentation in structures with in-line deduplication and quantified impact of fragmentation because of inter-version deduplication on re- save pace. The trouble is pretty excessive, and counting on backup tendencies also can bring about restore speed drop of more than $50 \%$. Furthermore, it affects modern-day backups the maximum, which can be also the maximum likely to be restored.

To address the hassle, we've got got got used the. This set of guidelines identifies the sparse boxes and rewrites them. It completes this gadget with the assist of historic records. The brand new set of regulations improves the restore normal general overall performance. The opportunity set of rules used to is the Cask-reveal set of rules (CMA), this set of rules is used to lessen the metadata overhead of the garbage collection, we further propose a Cask-display algorithm (CMA) to discover valid packing containers in preference to valid chunks.

This paper concentrates on fragmentation because of the inter-model duplication interior one or extra backup set.

\section{REFERENCES}

1. C. Dubnicki, L. Gryz, L. Heldt, M. Kaczmarczyk, W. Kilian, P. Strzelczak, J. Szczepkowski, C. Ungureanu, and M. Wel- nicki. HYDRAstor: a Scalable Secondary garage. In rapid'09: courtroom docket times of the seventh USENIX convention on file and storage technology, pages 197-210, Berkeley, CA, the usa, 2009. USENIX affiliation.

2. T. Asaro and H. Biggar. Records De-duplication and Disk-to- Disk Backup systems: Technical and business enterprise corporation Considera- tions, 2007. The enterprise company approach institution.
3. "Restoring deduped records in deduplication systems,"http://searchdatabackup.Techtarget.Com/charac teristic/Restoringdedupeddata-in-deduplicationstructures, 2010.

4. A. Muthitacharoen, B. Chen, and D. Mazires. A lowbandwidth network report machine. In In court cases of the 18th ACM Symposium on working systems requirements (SOSP '01, pages 174-187, big apple, the massive apple, u.S., 2001. ACM.

5. H. Biggar. Experiencing data De-Duplication: improving overall performance and reducing capability requirements, 2007. The business company technique organization

6. F. C. Botelho, P. Shilane, N. Garg, and W. Hsu, "reminiscence inexperienced sanitization of a deduplicated garage device," in Proc. USENIX fast, 2013.

7. M. Lillibridge, ok. Eshghi, D. Bhagwat, V. Deolalikar, G. Trezise, and P. Camble, "Sparse indexing: huge scale, inline deduplication the usage of sampling and locality," in Proc. USENIX speedy, 2009.

8. D. Meister and A. Brinkmann, "dedupv1: improving deduplication throughput using strong united states of america drives (SSD)," in Proc. IEEE MSST, 2010.

9. W.C Preston, Backup \& recuperation. O’Reilly Media, Inc., 2006.

10. F. Guo and P. Efstathopoulos, "constructing a immoderate common regular average performance deduplication tool," in Proc. USENIX ATC, 2011.

11. X. Lin, G. Lu, F. Douglis, P. Shilane, and G. Wallace , “ Migratory compression: Coarse-grained information reordering to beautify compressibility," in proc USENIX speedy, 2012.

12. F. Guo and P. Efstathopoulos, "building a highperformance deduplication machine ," in Proc. USENIX ACT, 2011.

13. S. Quinlan and S. Dorward. Venti: a contemporary Technique to archival storage. In speedy'02: proceedings of the convention on record and garage generation, pages 89-one 0 one, Berkeley, CA, america, 2002. USENIX affiliation.

14. [14] F. C. Botelho, P. Shilane, N. Garg, and W. Hsu, "memory efficient sanitization of a deduplicated storage system," in Proc. USENIX speedy, 2013. [15] M Lillibridge, ok. Eshghi, D. Bhagwat, V. Deolalikar, G. Trezise, and P. Camble, "Sparse indexing: massive scale, inline deduplication the use of sampling and locality," in Proc. USENIX rapid, 2009. [16] D. Meister and A. Brinkmann, "dedupv1: improving deduplication throughput using robust u . S . A . Drives (SSD)," in Proc. IEEE MSST, 2010.

15. M. Kaczmarczyk, M. Barczynski, W. Kilian, and C. Dubnicki, "lowering effect of information fragmentation because of in-line deduplication,"in Proc. ACM SYSTOR, 2012. 